\title{
Transporte y mercado interno en Colombia: una contribución a un debate hasta ahora desconocido, 1928-1950
}

\author{
Transportation and Domestic Market in Colombia: \\ a Contribution to a Heretofore Unknown Debate 1928-1950
}

Sebastián Villarreal Romero

Economista y Magíster en Economía de la Pontificia Universidad Javeriana http://orcid.org/0000-0002-9076-4914 sebastianvilla23@hotmail.com

\section{Darío A. Ortiz Navarro}

Economista y Magíster en Economía de la Pontificia Universidad Javeriana http://orcid.org/0000-0002-8706-0289

darortiz29@outlook.com

Fecha de recepción: 25 de septiembre de 2015

Fecha de aceptación: 15 de enero de 2016

Sugerencia de citación: Ortiz, D. y Villarreal, S. (2016). Transporte y mercado interno en Colombia: una contribución a un debate hasta ahora desconocido, 1928-1950. tiempo\&economía, 3(1), 83-107, doi: http://dx.doi.org/10.21789/24222704.1096

\section{Resumen ${ }^{1}$}

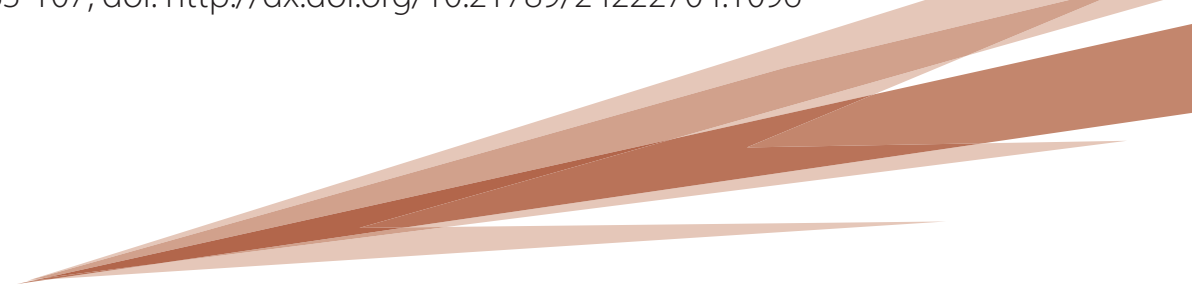

Algunos autores sostienen que durante el siglo XIX y principios del XX, el desarrollo del mercado interno colombiano fue escaso como consecuencia del atrasado sistema

1 Agradecemos el apoyo y los comentarios del profesor Edwin López Rivera. 
de transportes. Sin embargo, otros autores atribuyen el pobre desarrollo de las vías de comunicación precisamente a la ausencia de un mercado interno consolidado. El documento establece los términos de este debate inadvertido hasta ahora en la literatura sobre la primera mitad del siglo XX, incluso en la historiografía económica colonial, proponiendo la prueba de causalidad de Granger (procedimiento Toda-Yamamoto) como estrategia metodológica para contribuir empíricamente al debate.

Palabras clave: Transporte, mercado interno, causalidad.

Códigos JEL: C01, C12, N70, N76

\begin{abstract}
Some authors argue that during the nineteenth and early twentieth centuries, the development of the domestic market was limited as a result of the belated transportation systems. However, other authors attribute the poor development of communication paths, precisely by the absence of a consolidated domestic market. This paper sets out the terms of this unnoticed debate so far in the literature on the first half of the twentieth century, even in economic Colonial historiography, proposing the Granger Causality Test (Toda and Yamamoto procedure) as a methodological strategy to provide an empirical approach to the debate.
\end{abstract}

Keywords: Transportation, domestic market, causality.

JEL Codes: C01, C12, N70, N76 


\section{Introducción}

En este trabajo nos proponemos abordar la pregunta ¿Cuál fue la dirección de causalidad entre la expansión del sistema de transportes y el impulso del crecimiento del mercado interno colombiano a principios del siglo XX?

La pregunta nace de una inquietud intelectual que se deriva de la lectura de varios de los libros más populares en la enseñanza universitaria sobre la historia económica de nuestro país, en los que se encuentran explicaciones diferentes sobre la relación entre estas dos variables: el mercado interno y el sistema de transportes.

Por ejemplo, en el capítulo titulado "Las vicisitudes del modelo liberal (1850-1899)", de Melo (1991), en Historia económica de Colombia, compilado por Ocampo, se menciona el problema de los sistemas de transporte en el siglo XIX como la causa por la que "durante la época colonial y los primeros años de la república no había roto o reducido sustancialmente las barreras entre las diversas regiones" (Melo, 1991, p. 127). Para Melo, el bajo desarrollo del sistema de transportes contribuía a fragmentar el mercado interno. Pero esta idea, que hasta el día de hoy aparece como la tesis más fuerte, discrepa de las ideas de otros autores, quienes dan una explicación diferente: habría sido el pobre desarrollo del mercado interno la causa del subdesarrollo en los sistemas de transporte.

En otra obra reconocida en historia económica, Colombia y la economía mundial 1830-1910, de Ocampo (1984), se manifiesta el reconocimiento de las relaciones de producción y la baja productividad como factores determinantes del pobre desarrollo del mercado interno, $y$, debido a esto, en el sistema de transportes no hubo ningún progreso significativo, pues para qué esforzarse si no había una necesidad latente.

Dado el escaso desarrollo del mercado interno, el sistema de transportes necesario para satisfacer las necesidades de intercambio mercantil permaneció sumamente atrasado con relación a los patrones de la época. (Ocampo, 1984, p. 32)

Retomando la lectura de Melo se puede observar que él mismo reconocía la otra dirección: "la debilidad del comercio interregional tendía a reforzar las limitaciones del sistema de comunicaciones [...], resultaba difícil impulsar el desarrollo de carreteras y ferrocarriles que no parecían contar con carga suficiente para hacerlos rentables" (Melo, 1991, p. 127). Esta situación se presenta también en otros libros tanto de historiadores como de economistas. El lector puede remitirse al anexo A de este documento, donde se condensan algunas citas de varios de los autores sobre las distintas situaciones de causalidad que se presentan, ilustrando de esta forma el debate.

Pachón y Ramírez (2006, p. xv) sostienen que el estudio de la evolución de la infraestructura de transporte es importante para entender la dinámica de crecimiento de un país. Por su parte, Safford (2010, p. 523) afirma que el problema de los transportes puede ser el más fundamental en la historia económica del país. Aun cuando se enuncie el porqué de la importancia del tema, la verdadera motivación de esta investigación es la de aproximarse a una respuesta para esa inquietud que nació en un salón de clases y que no pudo solucionarse por falta de herramientas, pues en ese entonces se contaba solo con dos puntos de vista, era, o es, la palabra de unos contra la palabra de otros. 
Como lectores, descubridores y espectadores de este debate de ideas surge la necesidad de contribuir empíricamente, aprovechando los recursos que brindan recientes investigaciones como las de Ramírez (2006) y Greco (2004), quienes pusieron a disposición una nueva base de datos que permite llevar a cabo análisis empíricos aplicados a situaciones históricas.

Además de la motivación que da inicio a esta investigación, el documento resulta importante puesto que establece los términos de un debate historiográfico desconocido, y por la búsqueda de una respuesta al mismo que podrá contribuir a una mejor comprensión de la evolución de nuestros sistemas de transporte, en los que las decisiones de política se han mostrado en una sola dirección, como lo habrá de descubrir el lector más adelante. Posiblemente, este trabajo pueda contribuir también a la futura enseñanza de la historia del transporte en nuestro país.

Es así como el primer apartado del documento inicia presentando el sistema de transportes en el contexto latinoamericano, en cuya literatura abunda el impacto de los ferrocarriles sobre el comercio exterior, pero escasea en el caso de otros medios de transporte y respecto a su incidencia en los ámbitos locales. En este sentido, el trabajo también busca motivar futuros estudios sobre la relación del transporte con las economías domésticas. Posteriormente se establecen los términos de este debate historiográfico hasta ahora desconocido, recorriendo los primeros libros de historia económica del país.

La segunda parte se dedica a la contrastación empírica de las diferentes hipótesis, haciendo uso de la prueba de causalidad de Granger y abordando inicialmente el análisis para los ferrocarriles, seguido de uno que lo complementa con la inclusión de las carreteras. La tercera sección brinda algunas interpretaciones de los resultados desde el enfoque histórico. Finalmente, se presentan las conclusiones.

\section{Una aproximación al debate: entorno, alcance, estudios y limitaciones de los sistemas de transporte}

Es probable que el trabajo más destacado en el estudio de los transportes y su impacto sobre el crecimiento económico de un país sea el de Robert Fogel (1964). La importancia de este trabajo inspiró el de muchos otros que buscaron realizar un análisis aplicado al contexto latinoamericano, surgiendo de esta manera las contribuciones de Summerhill (1999), Herranz (2011), y para el caso colombiano, las de McGreevey (1975) y, posteriormente, las de Ramírez (1999, 2001, 2006, 2007), entre otros.

Las investigaciones latinoamericanas se concentraron en el impulso de los mercados ligados al desarrollo de los ferrocarriles, especialmente en México, Argentina y Brasil, que se destacan como los países con mayor cantidad de kilómetros de red ferroviaria construida. Argentina, incluso, tuvo niveles de longitud similares a los de algunos países europeos (Herranz et al., s. f.). Sin embargo, es de destacar que el primer país en Latinoamérica que abrió las líneas del ferrocarril fue Cuba, en 1837. Esto sucedió siendo todavía una colonia, algo sorprendente si se tiene en cuenta que, comparado con Gran Bretaña, la diferencia entre los primeros ferrocarriles fue de tan solo una década.

La construcción del ferrocarril en Latinoamérica se basó principalmente en el sistema de concesiones, y la falta de recursos públicos caracterizó su situación tanto en Brasil como en 
Colombia; esto trajo consigo el rezago internacional, pues muchos países desarrollados, como Estados Unidos, contaban con inversiones públicas significativas para su construcción.

Un caso especial dentro de la historia latinoamericana es el uruguayo. Siguiendo la hipótesis de Herranz et al. (s. f.), las redes férreas permitían unir las regiones apartadas, y, al ser Uruguay un país con una geografía diferente a la del resto del continente y con un tamaño inferior, las distancias cortas en los recorridos mermaban la incidencia que los ferrocarriles pudieran tener en el desarrollo económico y del transporte. A través de la historia se observa que Uruguay ha tenido una prioridad hacia las carreteras en lugar de los ferrocarriles, por las distancias más cortas de los desplazamientos, traducidas en menores costos, si se compara con los demás países latinoamericanos.

En términos generales, los trabajos que se refieren a los países latinoamericanos han presentado un énfasis en el impacto que tiene el transporte sobre el comercio exterior, por el carácter extractivo que representó, pero esta situación ha generado un vacío en cuanto a las economías locales.

Winston (1985) realizó un barrido de la literatura sobre economía del transporte y encontró que el énfasis se ha concentrado en el desarrollo conceptual de análisis de oferta y demanda. A pesar de que estos desarrollos han contribuido a un mejor entendimiento del tema, Winston cuestiona que los avances hayan permitido o permitan un cambio de política, señalando, además, que es importante explorar implicaciones más amplias del transporte en las economías locales. Al respecto, la investigadora Marta Herrera sostiene que en Colombia "los estudios se han interesado básicamente en el transporte y los intercambios culturales en relación con el comercio exterior por los puertos del Caribe, mas no en la situación del resto del país" (citada en Laurent, 2008, p. 245).

\section{El enfoque económico de los sistemas de transporte en Colombia}

En Colombia, la descripción de los sistemas de transporte ha sido un tema de creciente interés. Recientemente se han publicado varios trabajos históricos sobre ellos, ${ }^{2}$ pero el enfoque económico del transporte en el inicio no fue estudiado aisladamente sino como complemento de otros temas económicos generales.

Este enfoque económico de los sistemas de transporte empezó a destellar sus primeras luces en las observaciones del inglés William Wills y en los primeros libros de historia económica del país, donde se destacan las obras de Luis Eduardo Nieto Arteta (1942) y Luis Ospina Vásquez (1955).

Según Deas (1996a), Wills equiparaba en importancia el transporte con la educación de la población para el progreso del país. "Población para nuestras tierras y medios de transporte para nuestros frutos: estos son los verdaderos elementos del progreso; mientras no los tengamos no podremos ser verdaderos progresistas" (Wills, en Deas, 1996b, p. 173).

2 Véase Pachón y Ramírez (2006) para una descripción de los sistemas de transporte en Colombia y de su historia. Por su parte, Juan Santiago Correa (2010) ofrece amplia literatura sobre los ferrocarriles. 
Es notable también en las observaciones del señor Wills la poca relevancia que le dio a la geografía sobre los problemas de la Nueva Granada, diferenciándose así de otros autores. “Más que el aislamiento geográfico, Wills criticaba la falta de asociación que pudo haber reducido los efectos del mismo" (López, 2010, p. 26). En este punto, resulta importante entonces establecer el alcance de la geografía y la dispersión.

Desde el punto de vista de la nueva geografía económica, la geografía puede afectar el ingreso de un país por medio de dos canales: directos e indirectos. Los canales directos se enfocan en los efectos de la geografía sobre la productividad (naturales, climas extremos, esfuerzo físico). Pero la geografía también puede afectar el ingreso mediante canales indirectos, como la calidad de las instituciones.

Los defensores del canal indirecto subrayan la importancia de la geografía para el desarrollo, debido a sus efectos del pasado sobre la calidad institucional, al suponer que son perdurables. "En este contexto, las instituciones son buenas si limitan el alcance de la conducta depredadora del gobierno, actuando como catalizador para el establecimiento de los derechos de propiedad" (Rosas y Mendoza, 2004, p. 2).

En Colombia, las cordilleras de los Andes dividieron al país en tres regiones, pero con el paso del tiempo estas divisiones se convirtieron en políticas, étnicas y culturales. La minería caracterizó la economía de la región occidental (abastecimiento de regiones); en el oriente, la agricultura y la producción artesanal eran más fuertes. Por su parte, la costa Atlántica se destacaba por el comercio de bienes de consumo, el abastecimiento de otros productos y la importación de esclavos.

Siguiendo a Rosas y Mendoza (2004), en Colombia se presenta una gran diversidad regional, y esto implicaría también una diferencia en la eficiencia de las instituciones, por lo que la historia de Colombia sugiere que la geografía habría de influir por el canal indirecto, y las condiciones locales resultan fundamentales para la efectividad de los proyectos productivos. ${ }^{3}$

A su vez, Palacios y Safford (2002) hablan de una integración vertical en Colombia. Este tipo de integración hizo posible el comercio de alimentos entre regiones sin tener la necesidad de recurrir al comercio de larga distancia, "por tanto, si el clima y la topografía obstaculizaban el comercio interregional, también lo hacían superfluo" (Palacios y Safford, 2002, p. 26).

Estos mismos autores sostienen que tal situación podría haber apaciguado la necesidad de mejorar los sistemas de transporte por la falta de incentivos, pues no parecía necesario transportar bienes de intercambio entre las grandes regiones, precisamente como resultado de la complementariedad local, fruto de esa integración vertical.

En ese sentido, el debate en esta investigación se concentra solo entre las vías de comunicación terrestres y el mercado interno, suponiendo que los efectos que pudiera llegar a tener la geografía se encuentran inmersos en estas dos variables a través de las calidades institucionales.

3 Rosas y Mendoza (2004) estimaron los efectos de la geografía sobre el ingreso per cápita a nivel municipal en Colombia para 1999, utilizando la proporción de la población esclava en 1885 como variable instrumental de las instituciones vigentes. Los autores encontraron que la calidad institucional es determinante para el desarrollo económico y que, controlándola, algunas de las variables geográficas dejan de ser significativas sobre el ingreso de las personas, aunque otras sí tienen consecuencias significativas. 
Con relación a las obras pioneras de historia económica del país, en la de Nieto Arteta se encuentran diferentes puntos de vista de reconocidos autores sobre este debate, entre ellos Salvador Camacho Roldán y José María Samper.

Nieto (1975) menciona que en la memoria de 1872, don Salvador Camacho Roldan resaltó enérgicamente la imperiosa necesidad de las vías de comunicación:

Nuestra situación, escribió, envuelve un aislamiento tal que difícilmente podría ser apreciado en otro país. necesidad de las vías de comunicación, dice en otro lugar, es ya cuestión de ser o no ser. Hoy no se puede producir más que lo que se produce, por falta de salidas y de cambios. (En Nieto, 1975, p. 254)

Por otro lado, Nieto también cita a José María Samper, quien argumentaba que el predominio de la industria minera en la economía colonial ocasionaba varios hechos deplorables, dentro de los que se encuentra el poco desarrollo de las vías de comunicación:

La extracción exclusiva de metales preciosos, desdeñando la explotación del hierro y de los otros productos mineros, no fomenta el desarrollo de las vías de comunicación, ni el de las restantes industrias [...] Desgraciado el que no produce sino oro y piedras preciosas. (Samper, en Nieto, 1975, p. 249)

En la obra clásica de Luis Ospina Vásquez, Industria y protección en Colombia, se menciona sobre el tema que la preocupación de los gobernantes era la mejoría en la red que conectaba las principales regiones del país con el río Magdalena, principal vía de comunicación con el exterior.

Haciendo una revisión de literatura de trabajos más recientes, cobijando las obras comprendidas en la denominada Nueva Historia de Colombia y Nueva Historia Económica en Colombia, vale la pena resaltar que el debate, que se puede percibir desde los primeros trabajos de historia económica, también se trasladaría a los autores contemporáneos:

Durante el siglo XIX Colombia no tenía una economía nacional integrada. Y los altos costos del transporte limitaban los mercados en el ámbito regional. La falta de movimiento comercial restringía el mejoramiento del transporte, por disminuir los alicientes particulares y reducir los recursos fiscales. (Safford, 2010, p. 523. cursiva fuera del texto)

El desarrollo de la infraestructura de transporte afecta directamente el crecimiento económico de un país mediante la conexión de los mercados y la disminución del costo de transporte. Al reducirse éste, la sociedad ahorra recursos y los mercados se integran; esto a su vez genera economías de escala, reduce el diferencial de precios entre regiones y promueve tanto el comercio interno como externo. (Ramírez, 2006, p. xv. cursiva fuera del texto)

El debate, ${ }^{4}$ como lo demuestran las citas anteriores de Ramírez y Safford, continúa. Aunque la cita de Safford hace referencia al siglo XIX y la de Ramírez se escribe para la introducción del libro Economía colombiana del siglo XX, esta última sí resulta ser una afirmación general.

4 Podría parecer pretencioso decir que el debate no era conocido hasta el momento, si se quiere, pero al menos los términos del debate no habían sido establecidos hasta esta investigación. Véase el anexo A para una mejor ilustración sobre el debate. 
El considerar como cierta la afirmación según la cual el sistema de transportes impulsa el desarrollo del mercado interno ha llevado desde el siglo XIX a adoptar políticas encaminadas solo en este sentido, y no al contrario.

Se pueden citar varios casos: Tovar (1991, p. 109), por ejemplo, resume las propuestas de los consulados del comercio del siglo XIX, y el último punto de dichas propuestas menciona lo siguiente: "[...] impulsar las mejoras en las vías de comunicación, tan importantes para la disminución del costo final de las mercancías y los productos de todo género y para la ampliación del espacio mercantil" (Tovar, 1991, p. 109).

Así mismo, el dinero proveniente de la indemnización de Panamá puede ser un ejemplo, pues gran parte se invirtió en la infraestructura de transporte y no tuvo efectos fructíferos pues las obras no se realizaron con estudios técnicos ni económicos previos y no tenían propósitos determinados (Ramírez, 2007). Se construían con el único propósito de construir, y creyendo que esto tendría un impacto positivo en el mercado interno.

A su vez, para el siglo XX, Ramírez cita la promulgación de la ley 88 de 1931, de esta manera:

[...] en la ley 88 de 1931, se estableció por primera vez un plan de construcción concentrado en carreteras prioritarias y con un presupuesto asignado previamente. El gobierno consideró que una mayor inversión en carreteras tendría efectos benéficos sobre la economía porque, además de reducir los costos de transporte, ayudaba a integrar el mercado nacional. (Ramírez 2007, p. 398).

Estas políticas dan por sentado, o mejor, suponen que los efectos del transporte causarían mejoras en el mercado interno.

\section{Efectos hacia adelante: mercado interno vs. sistemas de transporte}

La Nueva Historia Económica clasifica dos efectos de la infraestructura de transporte: efectos hacia adelante y efectos hacia atrás. ${ }^{5}$ Los primeros, siguiendo a Summerhill (1999) para el caso particular del transporte, resultan del ahorro de costos en el transporte de productos o personas por ferrocarril u otro medio de transporte. Según Ramírez (2007), entre estos efectos se deben incluir los recursos que ahorra la sociedad por la reducción del precio de transporte y ampliación de los mercados.

Por su parte, los efectos hacia atrás se refieren a aquellos que inducen una demanda de los insumos necesarios para la construcción y el mantenimiento de los ferrocarriles.

Para determinar la dirección de causalidad entre el sistema de transportes y el mercado interno se hace necesario emplear el enfoque de los efectos hacia adelante. Sin embargo, cabe anotar que este trabajo no pretende estimar nuevamente un ahorro social, pues además de no ser una idea original, ya ha sido estimado para Colombia en dos ocasiones, corrigiendo

5 Esta terminología proviene de Albert O. Hirschman, quien acuño los términos enlaces o eslabonamientos, inicialmente, a los criterios para la planeación de inversiones en proyectos de desarrollo, definiéndolos "como una secuencia característica, más o menos imperativa, de decisiones de inversión que ocurren en el curso de la industrialización y, en términos más generales, del desarrollo económico" (en Furió-Blasco, 1998, p. 33). 
en la segunda (Ramírez, 1999) las debilidades o discusiones de la primera (McGreevey, 1971). Tampoco resulta conveniente para el objetivo de esta investigación adoptar alternativas diferentes a las del ahorro social para evaluar el impacto de la infraestructura, pues este tipo de metodologías no consideran el hecho de que muchos de los ferrocarriles fueron construidos con base en modelos económicos extractivos que reforzaban la orientación exportadora. Estas interpretaciones son conocidas bajo el nombre de literatura dependentista. Investigadores del transporte en América Latina reconocen que, a pesar de la importancia de estas interpretaciones, estos aspectos se encuentran fuera del alcance de sus investigaciones (Herrans, 2011).

Esta investigación se ajusta más a la idea de Coatsworth (1984, p. 18), según la cual "existe un eslabonamiento hacia adelante, por ejemplo, cuando los ferrocarriles proporcionan un insumo necesario (transporte) a otras unidades económicas". Esta definición deja a un lado el supuesto que ha impedido una visión más amplia del tema y que distingue este análisis del análisis de otros autores como Ramírez $(2001,2006,2007)$, al suponer que los costos de transporte son la única causa de los diferenciales de precios de un mismo bien entre regiones. Y es que Ramírez interpretó la pregunta - ¿El desarrollo en la infraestructura de transporte ha contribuido a la integración de un mercado nacional?- como si las reducciones en los costos de transporte explicaran reducciones en la divergencia de los precios agrícolas entre las diversas regiones colombianas. Su análisis concluye que, en cuanto a la integración, hay una limitación del mercado interno colombiano por la falta de una adecuada infraestructura de transporte. Pero es la misma autora quien reconoce en un pie de página lo siguiente: "por supuesto, los costos de transporte no son la única causa de los diferenciales de precios de un mismo bien entre regiones, también pueden influir otros factores" (Ramírez, 2006, p. 164). Esta circunstancia resulta provechosa para el objeto de este estudio al tratar de identificar parte de esos "otros factores".

El presente análisis de causalidad se llevará a cabo con la prueba de causalidad de Granger, siguiendo el procedimiento de Toda y Yamamoto (1995), y se abordará en dos partes. La primera estudia la relación entre el mercado interno y los ferrocarriles, y la segunda complementará el análisis con las carreteras, para así evaluar las diferentes hipótesis sobre la relación entre el Sistema de transportes (ST) y el mercado interno (MI). En el anexo A se muestra un cuadro comparativo de los autores que apoyan las diferentes distinciones de causalidad, que se resumen así:

- Causalidad unidireccional de MI hacia ST (MI $\longrightarrow$ ST);

- Causalidad de ST hacia MI (MI $\longleftarrow$ ST);

- Causalidad bilateral (MI $\longrightarrow$ ST);

- Independencia, cuando los conjuntos de coeficientes de MI y ST no son estadísticamente significativos. 


\section{Los datos}

Gracias a las recientes investigaciones de la economía colombiana en el marco de la Nueva Historia Económica, y en particular las investigaciones de Ramírez (1999, 2001, 2006, 2007), se encuentra disponible una importante base de datos que contribuye al estudio del desarrollo del transporte terrestre en Colombia, contenida en el CD ROM anexo estadístico del libro $L a$ infraestructura de transporte en Colombia durante el siglo XX (Pachón y Ramírez, 2006). De esta base se obtuvieron los datos del total de carga transportada por los ferrocarriles y la longitud de las redes férreas y de las carreteras. Los demás datos utilizados en el análisis que se presenta a continuación se obtuvieron de Greco (2004).

\section{Los ferrocarriles}

En la historia de Colombia han existido varios enfoques para estudiar el tema de los ferrocarriles: por una parte se encuentran trabajos de tipo empresarial, y por otro, su impacto en el desarrollo económico. Independiente de su enfoque, los autores coinciden en afirmar que el ferrocarril, o mejor, que sus rutas fueron diseñadas para articular productos primarios con el comercio exterior (Bejarano, 1991; Correa, 2010; McGreevey, 1975; Ramírez, 2001, 2006, 2007; Tirado, 1987, entre otros).

Solo un pequeño porcentaje del valor transportado por ferrocarriles correspondía al comercio interno colombiano, "lo que configuraba un sistema centrífugo de la infraestructura de transportes que apenas marginalmente vinculaba los mercados internos" (Bejarano, 1991, p. 196). El dinero proveniente de la indemnización de Panamá y los empréstitos se orientó a las obras públicas, y el gasto público nacional total pasó de 16,2\% en 1923 a 53,9\% en 1928. En este año, los gastos se destinaron a las vías de comunicación, básicamente a los ferrocarriles, que absorbieron más del $60 \%$ de las inversiones públicas del país, que trataba de orientar la construcción de las vías a crear y facilitar el mercado interno, promoviendo una red de tendencias centrípetas, en lugar de las centrifugas que predominaban hasta entonces (Bejarano, 1991).

Con base en lo anterior, y dado que a partir de la década de los cincuenta "el 50 por 100 de los ferrocarriles fueron clausurados y los restantes nacionalizados, por lo que el transporte de carga por tren perdió participación dentro del total de carga terrestre" (Ramírez, 2001, p. 105), el análisis se limitará al periodo comprendido entre 1928 y 1950.

La prueba de causalidad de Granger se utiliza para contrastar la hipótesis de Melo (1991), según la cual los sistemas de transporte no parecían contar con carga suficiente para su rentabilidad. De los resultados, que se muestran en el cuadro 1, se concluye que se puede rechazar la hipótesis nula según la cual no hubo causalidad de la longitud férrea a la carga transportada. En otros términos, la ampliación de la red férrea pudo haber ayudado a explicar la cantidad transportada por los ferrocarriles, pero no al contrario. 


\section{Cuadro 1. Prueba de Causalidad de Granger. Procedimiento Toda-Yamamoto. Longitud ferrocarriles vs. carga total transportada por ferrocarriles*}

\begin{tabular}{|c|c|c|}
\hline Hipótesis nula & $\Pi^{2}$ & Valor de probabilidad \\
\hline $\begin{array}{c}\text { Longitud de ferrocarriles no causa carga } \\
\text { transportada en el sentido Granger }\end{array}$ & 3,737 & 0,053 \\
\hline $\begin{array}{c}\text { Carga transportada no causa longitud de } \\
\text { ferrocarriles en el sentido Granger }\end{array}$ & 0,759 & 0,384 \\
\hline
\end{tabular}

* Se utilizó la prueba de raíz unitaria Dickey Fuller aumentada para determinar la estacionalidad de las series y se encontró que la variable que representa los ferrocarriles no es estacionaria, y la que representa la carga sí lo es. Por esta razón, se empleó el procedimiento Toda Yamamoto. Véase el anexo B.

Fuente: cálculos de los autores.

Sin embargo, como se ha mencionado a lo largo de este documento, gran parte de la carga transportada por los ferrocarriles se destinaba a las exportaciones, por lo que los resultados obtenidos no podrían estar reflejando exclusivamente el comportamiento del mercado interno.

En un principio se pensó, para mitigar este efecto, en sustraer del total de carga transportada por los ferrocarriles, las toneladas exportadas de café, el principal producto del país. ${ }^{6}$ Pero este ejercicio no podría generar resultados confiables, ya que no toda la carga cafetera con destino al exterior se transportaba por los ferrocarriles; además, se estarían dejando por fuera los demás productos exportables que los ferrocarriles transportaban. Las anteriores dificultades no permitirían explicar con seguridad la relación entre las variables que se quieren estudiar.

\section{Los ferrocarriles y las carreteras}

En la década de los treinta, con el ascenso del Partido Liberal al poder, se dio un cambio de énfasis hacia el sistema de carreteras, y se abandonó de esta forma el propósito anterior de construir un sistema nacional de ferrocarriles (McGreevey, 1975; Pachón y Ramírez, 2006; Latorre y Rincón, 1981; Bejarano, 1991). Resulta entonces pertinente tener en cuenta no solo el transporte férreo sino también el transporte por carreteras.

A pesar de tener disponibles los datos del total de carga transportada por los ferrocarriles, no existen datos confiables de la carga transportada por carreteras, lo que se convierte en una gran limitación, dado el papel preponderante de las carreteras a partir de la década de los treinta.

Con esta limitante, y atendiendo a la definición general del Producto Interno Bruto, se plantea la idea de emplear los datos del PIB de Greco (2004) y mitigar el mercado externo con la sustracción de las exportaciones totales del PIB para el periodo estudiado, las cuales

6 El café fue el principal producto de exportación de Colombia hasta 1986. Los datos provienen del CD ROM anexo estadístico de Greco (2004).

7 En millones de pesos de 1975.

tiempo\&economía

Vol. 3 N $^{\circ} 1$ - I semestre de 2016 
registraron una participación promedio de 23,14\% entre 1928 y 1950, pasando de representar un $25,90 \%$ a un $17,51 \%$, respectivamente. Así, se utiliza la prueba de Granger para verificar la posible existencia de una relación de causalidad y determinar la dirección entre la expansión de los sistemas de transporte - representada por la suma de la longitud total de los ferrocarriles más la longitud de carreteras en Colombia ${ }^{8}$ - y la expansión del mercado interno, tomando como referencia el Producto Interno Bruto menos las exportaciones de cada uno de los años del periodo. ${ }^{9}$

Cuadro 2. Prueba de Causalidad de Granger. Procedimiento Toda- Yamamoto. Mercado interno vs. sistema de transportes*

\begin{tabular}{|c|c|c|}
\hline Hipótesis nula & $\square^{2}$ & Valor de probabilidad \\
\hline $\begin{array}{c}\text { Sistema de transportes no causa mercado } \\
\text { interno en el sentido Granger }\end{array}$ & 0,009 & 0,921 \\
\hline $\begin{array}{c}\text { Mercado interno no causa sistema de trans- } \\
\text { portes en el sentido Granger }\end{array}$ & 2,723 & 0,098 \\
\hline
\end{tabular}

* En este caso, la prueba ADF indica también un orden de integración diferente entre las series. Véase el anexo C. Fuente: cálculos de los autores

En el cuadro 2 se presentan los resultados, en los que se rechaza la hipótesis nula de no causalidad del mercado interno hacia sistema de transportes para un nivel de significancia del $10 \%$. De aquí que el mercado interno pudo haber ayudado a explicar el sistema de transportes pero el sistema de transportes no ayudó a explicar el mercado interno. En el próximo apartado se expondrán los resultados con las interpretaciones que ofrece la historia.

\section{Los resultados empíricos y la historia}

\section{Los ferrocarriles}

La posible causalidad de los ferrocarriles hacia la carga transportada confirma las hipótesis tradicionales de los historiadores y economistas sobre el "sistema centrífugo" de la infraestructura férrea. También refuerza trabajos empíricos como el de Ramírez (2006, p. 45), quien demostró que "el transporte de carga por ferrocarriles es elástico al cambio de la longitud de la red".

$8 \mathrm{ST}=$ (total $\mathrm{km}$ de ferrocarriles + total $\mathrm{km}$ carreteras construidas)

$9 \mathrm{Ml}=(\mathrm{PIB}-\mathrm{X})$ 
El problema de analizar solamente los ferrocarriles es que estos se construyeron relativamente tarde, cuando ya las carreteras se construían paralelamente a la red. El camión, como medio de transporte alternativo, permitía menor tiempo de desplazamiento. Esto puede explicar por qué, pese a los incentivos de carga, estos no se ven reflejados en la extensión de la red.

Según Salazar (2000), el primer camión llegó a Colombia a principios de 1917:

La introducción del camión en Colombia alteró todo lo referente al transporte porque el río y el ferrocarril perdieron su supremacía, y del aproximadamente $75 \%$ de las cargas que transportaban en 1925 se había pasado en 1956 a un insignificante 8,3\% del primero y apenas a un $20,7 \%$ del segundo. Para entonces los camiones movilizaban el $69,5 \%$ de la carga y los grandes volúmenes utilizaban las carreteras por donde ya en 1951 transitaban más de siete mil camiones, con capacidad para cinco toneladas cada uno. (Salazar, 2000, p. 96)

\section{Los ferrocarriles y las carreteras}

Para entender el supuesto de causalidad del mercado interno hacia el sistema de transportes, que se deriva del rechazo de la hipótesis de negación, es importante recordar los acontecimientos más relevantes durante la primera mitad del siglo XX.

Según las cifras de GRECO (2004), entre 1928 y 1950, el crecimiento promedio anual del producto interno bruto fue del 3,76\%. Esta cifra aumenta a 4,1\% si no se tienen en cuenta las exportaciones en el cálculo, aproximándose a una representación del mercado interno colombiano. A inicios de este periodo, la población del país bordeaba los ocho millones, y en la mitad del siglo XX superaba los doce millones. Entre tanto, el PIB per cápita registró un crecimiento promedio anual de 1,5\%.

El mercado interno se caracterizó por el cambio sectorial de la agricultura a la industria y a los servicios, sectores en los que la productividad es mayor (Urrutia y Posada, 2007). Aunque para algunos autores este crecimiento significativo en la industria manufacturera no fue suficiente como para hablar de industrialización en Colombia.

Este cambio sectorial pudo contribuir también a un cambio de modelo económico tradicional en Colombia, al que José Antonio Ocampo (1984) bautizó como producciónespeculación. El modelo, que consistía en aprovechar las circunstancias extraordinarias que ofrecían los mercados en desequilibrio, y en el que no había interés por mejorar las técnicas ni relaciones de producción, es utilizado por Ocampo para explicar el comercio exterior del siglo XIX. Cuando se lee a Camacho Roldán (1892) se puede percibir la idea del modelo producciónespeculación en su discurso, al abordarlo no solamente como una característica de nuestra política de comercio exterior sino también como una muestra de nuestra situación interna: "Si de los cultivos de exportación pasamos a nuestras cosechas de consumo interior, doloroso es decir que no encontramos compensación alguna en el cambio de investigación" (Camacho Roldán, 1892, p. 640). 
El cambio importante hacia otros sectores más productivos estuvo relacionado con las políticas proteccionistas iniciadas en 1931, comúnmente conocidas como "sustitución de importaciones", que motivaron los incentivos necesarios para producir aquellos bienes que antes solo se obtenían mediante las importaciones. De acuerdo con Montenegro y Ocampo (2007), las medidas favorecieron especialmente la producción doméstica de alimentos, tejidos de lana, cemento y cerveza.

Lo anterior contribuyó a que el mercado interno tuviera un mayor dinamismo y generó la necesidad de construir vías de comunicación, dando una posible explicación a la dirección de causalidad del mercado interno hacia los sistemas de transporte. Adicionalmente, la forma como se está entendiendo el mercado interno en este contexto también explicaría los resultados pues, a diferencia de los ferrocarriles, las carreteras fueron construidas con dineros públicos.

Gráfico 1.

Longitud red férrea vs. carreteras $(\mathrm{km})$

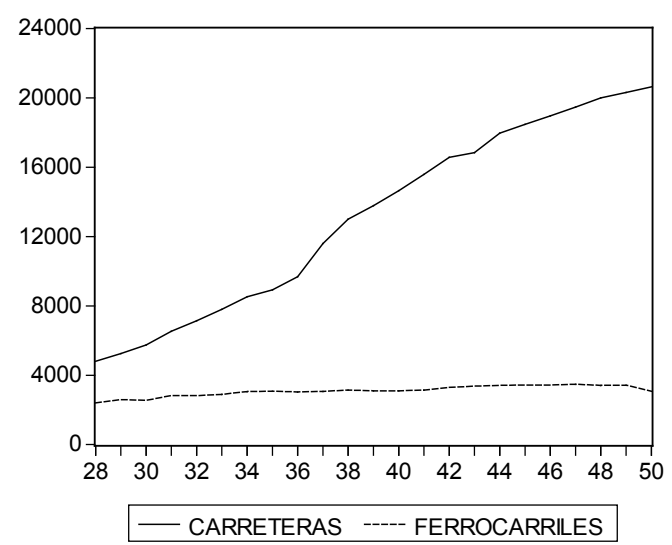

Grafico 2.

PIB menos exportaciones (en millones de pesos)

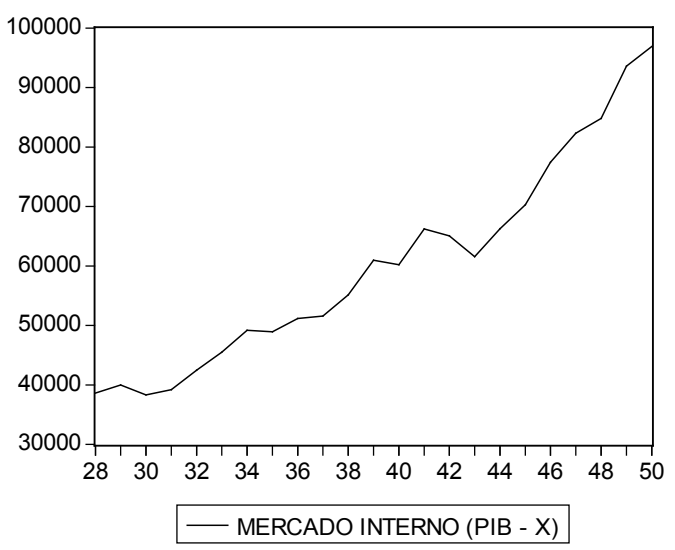

Fuente: CD ROM anexo estadístico, Ramírez (2006). Fuente: CD ROM anexo estadístico, Greco (2004).

El gráfico 1 representa la longitud de las redes férreas y de las carreteras, que muestra una tendencia creciente de las carreteras y un estancamiento de los ferrocarriles. La variable utilizada como mercado interno también muestra una tendencia creciente con algunos altibajos, el más notable durante la Segunda Guerra Mundial (ver el gráfico 2).

Quizás, el cambio más importante del periodo haya sido el que este crecimiento industrial acelerado y sostenido causó en la estructura de la economía colombiana, pasando el sector industrial de representar el 8,9\% del PIB en 1929 a casi doblarse en 1945 , con el $16 \%$ de toda la producción. (Brando 2010, p. 205).

Ahora entendamos, desde las razones históricas, los motivos por los cuales el sistema de transportes no causó el mercado interno ${ }^{10}$.

10 Dada la manera de representación del mercado interno que se utilizó, puede ser más preciso interpretar los resultados en términos de crecimiento, y no tanto así como de conexión. 
En primer lugar, las redes férreas estaban desarticuladas, y se evidencia una esparcimiento casi sin conexión, lo que dificultaba la integración y consolidación de un mercado interno, sumado a un factor técnico que incluso imposibilita conectarlas hoy en día, si se quisiera hacerlo, y es que las líneas fueron creadas con diferentes especificaciones técnicas: unas son más anchas que otras, y no todas las máquinas las pueden recorrer. "En la mayoría de los ferrocarriles de la región occidental era de una yarda, mientras que en los ferrocarriles de la zona oriental era de un metro" (Ramírez, 2006, p. 50).

En cuanto a las carreteras, debe decirse que, a pesar de las buenas intenciones que planteaba la ley 88 de 1931 —en la que se suponía que la construcción de carreteras ayudaría a integrar (más) el mercado nacional, cuya hipótesis es utilizada por varios de los historiadores-, finalmente las metas propuestas en la ley no se cumplieron (en parte por la recesión económica entre 1930 y 1934 y la política restrictiva de finales de esta misma década). Pese a esto, cabe señalar que sí es cierto que la construcción de carreteras aumentó durante la primera mitad del siglo XX. Ahora bien, esto no significa que más es mejor. Veamos.

A diferencia de los ferrocarriles, las carreteras no se construyeron mediante el sistema de concesiones, y se construyeron entonces con dineros públicos. ${ }^{11}$ Muchas de las carreteras se construyeron con propósitos transitorios, incluso, varias de ellas solo se utilizaron para transportar rieles durante la construcción de las carrileras, y, una vez terminado este trabajo, las carreteras no tuvieron el mantenimiento necesario y fueron abandonadas, al no ser posible encontrar una alternativa para su utilización, debido al carácter transitorio que tenían (Ramírez, 2006).

Otro factor en que Ramírez (2006) concentra su atención se refiere a las pujas regionalistas entre los políticos, lo que llevó a la atomización de los recursos que se invertían. La repartición exacerbada de recursos para satisfacer a los congresistas impidió en muchos casos que las carreteras se terminaran de construir por falta de presupuesto. Además, el Estado otorgaba generosos subsidios a los departamentos para la construcción de carreteras, lo que generó incentivos perversos por construir a costos muy bajos, en detrimento de la calidad de las carreteras; de esta manera se podría acceder y aprovechar el subsidio por estas construcciones de pésima calidad. Como resultado, el valor de los subsidios era, de lejos, mucho más alto respecto a los costos de su construcción. Más que un incentivo, el subsidio se convirtió en un negocio.

El regionalismo también fue motivo de desintegración física, pues los recursos se concentraban para favorecer solo obras locales, y nunca se pensó en un mercado nacional. Estos hechos confirman el supuesto del canal indirecto de la geografía, por medio de las instituciones que permanecen a través de la historia.

El caos legislativo se tradujo en la inapropiada asignación de recursos, la mala calidad de las vías y la construcciones de una gran número de carreteras poco relevantes, dejando de lado la construcción de vías prioritarias para el desarrollo económico del país [...] además, la mayoría de las carreteras se construían sin ningún plan ni estudio técnico, lo que se tradujo en vías aisladas e inconclusas que a la larga no prestaban ningún servicio. (Ramírez, 2006, p. 63)

11 Con la ley 27 de 1909 se prohibió la construcción de carreteras por concesiones. 
Los anteriores acontecimientos, que se refieren a la década de los treinta del siglo XX, son un espejo de lo que ocurrió en el siglo XIX. Dicha circunstancia valida, nuevamente, la hipótesis de la perdurabilidad de las instituciones:

Durante casi todo el siglo XIX los políticos colombianos enfrentaron el problema de las prioridades haciendo caso omiso de él. Como resultado, proliferaron los proyectos pequeños mal financiados y muchas veces fallidos y no hubo mayor avance en el transporte terrestre [...] (Palacios y Safford, 2002, p. 27)

Estos hechos pueden ilustrar el porqué de los resultados, en los que el mercado interno pudo haber causado el sistema de transportes, pero el sistema de transportes no causó el mercado interno en la primera mitad del siglo XX. Al respecto, Muriel (2009) comenta que la ideologización del crecimiento interno, vía medios de transporte, hizo que desde el Período de la Danza de los Millones ${ }^{12}$ se creyera que los ferrocarriles traerían consigo el desarrollo capitalista de la Nación; de acuerdo con esta investigación, la idealización también compete a las carreteras, en fin, al sistema de transportes.

\section{Conclusiones}

Los resultados indican una posible relación de causalidad unidireccional del mercado interno hacia el sistema de transportes. El mercado interno propició los incentivos necesarios para la expansión del sistema de transportes como resultado de la política de sustitución de importaciones iniciada en 1931, acaparando la demanda de los bienes que anteriormente se comercializaban con el exterior; a esto se suma el cambio sectorial que presentó la economía del país de la agricultura a la industria y los servicios. Pese a lo anterior, el sistema de transportes no reforzó esta situación. Los ferrocarriles fueron diseñados para el comercio exterior; las carreteras se construyeron sin estudios técnicos, y los regionalismos, representados por los dirigentes políticos, generaron una situación en la que solo se buscaba el interés regional y no se pensaba en un mercado nacional.

El análisis, sin embargo, no permite establecer quién o quiénes de los autores citados en el anexo A tienen la razón, por cuanto varias de sus consideraciones se refieren al siglo XIX. Es posible que las diferencias en las interpretaciones de los autores sobre la relación entre el sistema de transportes y el mercado interno se deban a la atención que prestaron, ya sea a lo normativo o a lo positivo. Por ejemplo, las interpretaciones de un análisis basado en la lectura de los proyectos de ley o de las leyes en sí pueden diferir respecto a las de un análisis basado en cifras. Dicha situación se reconoce como "La Máxima de Hume".

Para futuros estudios sería interesante abordar un análisis contrafactual, ya que, a partir de los resultados de esta investigación, se puede pensar que un mercado interno más grande y dinámico de lo que realmente fue hubiera permitido un mejor desarrollo del sistema de transportes.

12 El término hace referencia al dinero proveniente de la indemnización por Panamá. En total, 25 millones de dólares que se pagaron entre 1923 y 1926. 
Esta es una contribución empírica al debate aquí manifiesto, enmarcándose así dentro de la Nueva Historia Económica o Cliometría, con la esperanza de retomar la atención de los profesionales de la historia y de la economía, quienes podrán aportar no solo con sus futuras investigaciones sino también con su forma de enseñanza.

\section{Referencias}

Bejarano, J. A. (1991). El despegue cafetero (1900-1928). En J. A. Ocampo (comp.), Historia Económica de Colombia (pp. 173-206). Bogotá: Siglo XXI.

Brando, C. (2010). La industrialización a medias. En S. Kalmanovitz (ed.). Nueva Historia Económica de Colombia. Bogotá: Taurus. pp. 197-213

Camacho Roldán, S. (1892). Escritos varios. Vol. 2. Bogotá: Librería Colombiana.

Coatsworth, J. H. (1984). El impacto económico de los ferrocarriles en el porfiriato: Crecimiento contra desarrollo. México: Ediciones Era.

Correa, J. S. (2010). Inversión extranjera directa y construcción de ferrocarriles en Colombia: El caso del ferrocarril de Panamá (1849-1869). Estudios Gerenciales, 26(115), 141-160.

Deas, M. (1996a). Vida y opiniones de Mr. William Wills, tomo I. Bogotá: Tercer Mundo.

Deas, M. (1996b). Vida y opiniones de Mr. William Wills, tomo II. Bogotá: Tercer Mundo.

Ferro, G. (1994). A lomo de mula. Bogotá: Fondo Cultural Cafetero.

Fogel, R. (1964). Railroads and american economic growth. Essays in econometric history. Baltimore: The Johns Hopkins University Press.

Furió-Blasco, E. (1998) (comp.). Albert O. Hirschman y el camino hacia el desarrollo económico. Una antología de ensayos anteriores a "la estrategia del desarrollo económico". México: FCE.

Granger, C. W. J. (1969). Investigating causal relations by econometric models and Crossspectral methods. Econometrica, 37(3), 424-438.

Greco (Grupo de Estudios del Crecimiento Económico) (2004). El crecimiento económico colombiano en el siglo XX. Bogotá: FCE.

Herranz, A. (2011). The contribution of railways to economic growth in Latin America before 1914: A growth accounting approach. Universidad de Barcelona. Recuperado el 18 de septiembre de 2012 de http://mpra.ub.uni-muenchen.de/33578/

Herranz, A., Bignon, V. \& Esteves, R. (s. f.). The big push, railways and export growth in Latin America, 1865-1913. s. I.: s. e.

Latorre, J. R \& Rincón, J. (1981). El desarrollo del transporte férreo en la perspectiva del mercado interior 1850-1930. Tesis de Grado, Universidad de Antioquia, Medellín, Colombia.

Laurent, M. (2008). Contrabando en Colombia en el siglo XIX: Prácticas y discursos de resistencia y reproducción. Bogotá: Universidad de los Andes. 
López, E. (2010). Circuitos mercantiles de la ciudad de Santa Fe a finales de la época colonial. Tesis de Maestría, Universidad Nacional de Colombia, Bogotá. Recuperado el 18 de septiembre de 2012 de http://www.bdigital.unal.edu.co/2132/

McGreevey, W.P. (1971). An Economic History of Colombia: 1845-1930. Londres: Cambridge University Press.

McGreevey, W. P. (1975). Historia económica de Colombia: 1845-1930. Bogotá: Tercer Mundo Editores.

Melo, J. O. (1991). Las vicisitudes del modelo liberal (1850-1899). En J. A. Ocampo (comp.). Historia Económica de Colombia. Bogotá: Siglo XXI. pp. 119-172

Montenegro, A. \& Ocampo J.A, (2007). Crisis mundial, protección e industrialización. Cali: Editorial Norma.

Muriel, R. D. (2009). Colombia: comercio y transportes, 1850-1929. Medellín: Editorial Pi.

Nieto, L.E. (1942). Economía y cultura en la historia de Colombia. Bogotá: Librería Siglo XX.

Nieto, L. E. (1975). Economía y cultura en la historia de Colombia. Bogotá: Ediciones Tiempo Presente.

Ocampo, J. A. (1984). Desarrollo exportador y desarrollo capitalista colombiano en el siglo XIX. En Colombia y la economía mundial 1830-1910 (pp. 21-77). Bogotá: Siglo XXI.

Ospina, L. (1955). Industria y protección en Colombia, 1810-1930. Medellín: Editorial Santa Fé

Palacios, M. \& Safford, F. (2002). Colombia. País fragmentado, sociedad dividida. Su historia. Bogotá: Editorial Norma.

Ramírez, M. T. (1999). On infraestructure and economic growth. Ph.D. Dissertation, Universidad de Illinois.

Ramírez, M. T. (2001). Los ferrocarriles y su impacto sobre la economía colombiana. Revista de historia económica, XIX (1), 81-121.

Ramírez, M. T. (2006). Desarrollo de la infraestructura de transportes en Colombia: 1900-1950. En A. Pachón \& M. T. Ramírez, La infraestructura de transporte en Colombia durante el siglo XX. (pp. 3-224) Bogotá: FCE.

Ramírez, M. T. (2007). Efectos de eslabonamiento de la infraestructura de transporte sobre la economía colombiana: 1900-1950. En J. Robinson \& M. Urrutia (eds.), Economía colombiana del siglo XX. Un análisis cuantitativo (pp. 383-457). Bogotá: FCE.

Rosas A. \& Mendoza, J. (2004). The economic effects of geography: Colombia as a case study. Documentos de Economía. No. 11 (junio 2004). Pontifica Universidad Javeriana.

Safford, F. (2010). El problema de los transportes en Colombia en el siglo XIX. En A. Meisel, \& M. T. Ramírez (eds.), Economía colombiana del siglo XIX. Bogotá: FCE. pp. 523-573

Salazar, J. (2000). De la mula al camión. Apuntes para una historia del transporte en Colombia. Bogotá: Tercer Mundo Editores.

Samper, D. (1965). Los transportes en Colombia. Bogotá: Contraloría General de la República. 
Summerhill, W. R. (1999). Los avances del transporte y el crecimiento económico en Brasil y México. En S. Haber (ed.), Cómo se rezagó la América Latina: Ensayos sobre las historias económicas de Brasil y México, 1800-1914. México: FCE. pp. 115-144

Tirado, A. (1987). Introducción a la historia económica de Colombia. Bogotá: El Áncora Editores.

Toda, H. \& Yamamoto, T. (1995). Statistical inferences in vector autoregressions with possibly integrated processes. Journal of Econometrics, 66, 225-250.

Tovar, H. (1991). La lenta ruptura con el pasado colonial (1810-1850). En J. A. Ocampo (comp.), Historia Económica de Colombia. Bogotá: Siglo XXI. pp. 87-116

Urrutia, M. \& Posada, C. E. (2007). Un siglo de crecimiento económico. En J. Robinson \& M. Urrutia (eds.), Economía colombiana del siglo XX. Un análisis cuantitativo. Bogotá: FCE. pp. 11-33

Vásquez, M. (2012). Economía política en la obra de Salvador Camacho Roldán. Bogotá: Universidad Externado de Colombia.

Winston, C. (1985). Conceptual development in theeconomics of transportation: An interpretive survey. Journal of Economic Literature, XXIII, 57-94. 


\begin{tabular}{|c|c|c|}
\hline \multicolumn{3}{|c|}{ Anexo A. Distinción de causalidad según autores } \\
\hline $\mathrm{MI} \longrightarrow \mathrm{ST}$ & $\mathrm{Ml} \triangleleft \mathrm{ST}$ & $\mathrm{Ml} \rightleftarrows \mathrm{ST} / \mathrm{Independencia}$ \\
\hline $\begin{array}{l}\text { Frank Safford (2010, p. 523): La falta } \\
\text { de movimiento comercial restringía el } \\
\text { mejoramiento del transporte, por disminuir } \\
\text { los alicientes particulares y reducir los } \\
\text { recursos fiscales. }\end{array}$ & $\begin{array}{l}\text { María Teresa Ramírez }(2006, p . x v) \text { : El } \\
\text { desarrollo de la infraestructura de transporte } \\
\text { afecta directamente el crecimiento } \\
\text { económico de un país mediante la conexión } \\
\text { de los mercados y la disminución del costo } \\
\text { de transporte. }\end{array}$ & $\begin{array}{l}\text { Jorge Orlando Melo (1991, p. 127): El } \\
\text { desarrollo del sistema de transportes no } \\
\text { había roto o reducido sustancialmente las } \\
\text { barreras entre las diversas regiones }[. . .] \text { los } \\
\text { elevados costos del transporte reducían la } \\
\text { movilización... }\end{array}$ \\
\hline $\begin{array}{l}\text { José María Samper (en Nieto 1975, p. 249): La } \\
\text { extracción exclusiva de metales preciosos, } \\
\text { desdeñando la explotación del hierro y de } \\
\text { los otros productos mineros, no fomenta el } \\
\text { desarrollo de las vías de comunicación, ni el } \\
\text { de las restantes industrias [...] Desgraciado } \\
\text { el que no produce sino oro y piedras } \\
\text { preciosas. }\end{array}$ & $\begin{array}{l}\text { Salvador Camacho Roldán (en Nieto, } \\
\text { 1975, 254): Nuestra situación, envuelve un } \\
\text { aislamiento tal, que difícilmente podría ser } \\
\text { apreciado en otro país. La necesidad de las } \\
\text { vías de comunicación es ya cuestión de ser } \\
\text { o no ser [...] ya no se puede producir más } \\
\text { que lo que se produce, por falta de salidas } \\
\text { y de cambios. }\end{array}$ & $\begin{array}{l}\text { Al mismo tiempo, la debilidad del } \\
\text { comercio interregional tendía a reforzar las } \\
\text { limitaciones del sistema de comunicaciones } \\
{[\ldots] \text {, resultaba difícil impulsar el desarrollo }} \\
\text { de carreteras y ferrocarriles que no parecían } \\
\text { contar con carga suficiente para hacerlos } \\
\text { rentables. }\end{array}$ \\
\hline $\begin{array}{l}\text { Juan Santiago Correa (2010, p. 144): El } \\
\text { tam la rentabilidad financiera y social de } \\
\text { proyectos más ambiciosos fue así como se } \\
\text { construyeron redes desarticuladas }[. . .]\end{array}$ & $\begin{array}{l}\text { Álvaro Tirado Mejía (1987, p. 219): La } \\
\text { carencia de vías de comunicación en el } \\
\text { interior del país fue un obstáculo tremendo } \\
\text { para el desarrollo de un mercado interno } \\
\text { [...]Tales condiciones de transporte inhibían } \\
\text { naturalmente el desarrollo de un mercado } \\
\text { nacional. }\end{array}$ & $\begin{array}{l}\text { William Paul McGreevey }(1975, \text { p. 252): A } \\
\text { pesar de las evidentes dificultades [en los } \\
\text { sistemas de transporte] tuvo lugar en el } \\
\text { país un comercio interno relativamente } \\
\text { dinámico. }\end{array}$ \\
\hline $\begin{array}{l}\text { José } \boldsymbol{A} \text {. Ocampo (1984, p. 32): Dado el escaso } \\
\text { desarrollo del mercado interno, el sistema } \\
\text { de transportes necesario para satisfacer } \\
\text { las necesidades de intercambio mercantil } \\
\text { permaneció sumamente atrasado con } \\
\text { relación a los patrones de la época. }\end{array}$ & $\begin{array}{l}\text { Jesús A. Bejarano (1991, p. 196): La principal } \\
\text { limitación de la economía nacional era la } \\
\text { carencia de un sistema articulado de medios } \\
\text { de transporte para los productos... }\end{array}$ & \\
\hline
\end{tabular}

Fuente: elaboración propia con base en las citas.

1 El tema de los transportes y, en general, la obra de McGreevey se enfocó principalmente en el impacto del comercio exterior sobre el desarrollo económico del país. Aun así, McGreevey menciona que los elementos estudiados fueron analizados aisladamente y sin tratar de integrarlos dentro de un modelo que los ubique en un esquema de causalidad. "La historiografía económica colombiana se encuentra en un estado muy primitivo que no permite emprender tal análisis cuidadosamente" (McGreevey, 1975, p. 14). 
Anexo B. Modelo que incluye longitud ferrocarriles $(\mathrm{km})$ y carga transportada por ferrocarriles (t), 1928-1950

Prueba Dickey Fuller aumentada (ferrocarriles)

\begin{tabular}{c|c}
\hline Estadístico ADF & $-\mathbf{0 , 6 0 3}$ \\
\hline & \\
Valor crítico al 1\% & $-4,440$ \\
Valor crítico al 5\% & $-3,633$ \\
Valor crítico al 10\% & $-3,255$ \\
\hline
\end{tabular}

Nota: no se puede rechazar la hipótesis nula de la existencia de raíz unitaria.

\begin{tabular}{c|c}
\hline Variable dependiente t-1 & $-0,148$ \\
Estadístico t & $(-0,603)$ \\
Constante & 516,186 \\
Estadístico t & $(0,815)$ \\
Tendencia & $-2,445$ \\
Estadístico t & $(-0,208)$ \\
Número de rezagos & 0 \\
\hline
\end{tabular}

Nota: el número de rezagos está determinado automáticamente por el criterio de selección Schwarz.

Prueba Dickey Fuller aumentada (carga-ferrocarriles)

\begin{tabular}{l|c}
\hline Estadístico ADF & $-\mathbf{3 , 2 6 4}$ \\
\hline & \\
Valor crítico al 1\% & $-4,441$ \\
Valor crítico al 5\% & $-3,633$ \\
Valor crítico al 10\% & $-3,255$ \\
\hline
\end{tabular}

Nota: para el 10\% de significancia se rechaza la Hipótesis nula de existencia de raíz unitaria.

\begin{tabular}{c|c}
\hline Variable dependiente t-1 & $-0,355$ \\
Estadístico t & $(-3,264)$ \\
Constante & 512248,9 \\
Estadístico t & $(2,191)$ \\
Tendencia & 60963,42 \\
Estadístico $t$ & $(3,839)$ \\
Número de rezagos & 0 \\
\hline
\end{tabular}

Nota: el número de rezagos está determinado automáticamente por el criterio de selección Schwarz. 
104 Transporte y mercado interno en Colombia: una contribución a un debate hasta ahora desconocido, 1928-1950

Criterios de selección para obtener el rezago óptimo

\begin{tabular}{cccc}
\hline Número de rezagos & Akaike & Schwarz & Hannan-Quinn \\
& & & \\
0 & 42,526 & 42,625 & 42,539 \\
1 & $39,638^{*}$ & $39.935^{*}$ & $39.679^{*}$ \\
3 & 40,014 & 40,508 & 40,082 \\
4 & 40,369 & 41,062 & 40,465 \\
5 & 40,200 & 41,090 & 40,323 \\
\hline
\end{tabular}

* Indica el rezago seleccionado según el criterio

Prueba Dickey Fuller aumentada D (ferrocarriles)

\begin{tabular}{ll}
\hline Estadístico ADF & $-4,756$ \\
\hline & \\
Valor crítico al 1\% & $-4,468$ \\
Valor crítico al 5\% & $-3,645$ \\
Valor crítico al 10\% & $-3,261$ \\
\hline
\end{tabular}

Nota: Para todos los niveles de significancia se rechaza la hipótesis nula de existencia de raíz unitaria en las primeras diferencias.

\begin{tabular}{cc}
\hline Variable dependiente t-1 & $-1,409$ \\
estadístico t & $(-4,756)$ \\
Constante & 168,296 \\
estadístico t & $(2,761)$ \\
Tendencia & $-10,445$ \\
Estadístico $t$ & $(-2,533)$ \\
Número de rezagos & 0 \\
\hline
\end{tabular}

Nota: el número de rezagos se determinó automáticamente por el criterio de selección Schwarz. 


\section{Anexo C. Modelo que incluye ST y MI, 1928-1950 \\ Prueba Dickey Fuller aumentada (MI)}

\begin{tabular}{cc}
\hline Estadístico ADF & $-1,091$ \\
Valor crítico al 1\% & $-4,441$ \\
Valor crítico al 5\% & $-3,633$ \\
\hline Valor crítico al 10\% & $-3,255$ \\
\hline Nota: no se puede rechazar la hipótesis nula de la existencia de raíz unitaria. \\
\hline Variable dependiente t-1 & $-0,175$ \\
Estadístico t & $(-1,091)$ \\
Constante & 5843,701 \\
Estadístico t & $(1,137)$ \\
Tendencia & 615,490 \\
Estadístico $t$ & $(1,550)$ \\
Número de rezagos & 0 \\
\hline
\end{tabular}

Nota: el número de rezagos se determinó automáticamente por el criterio de selección Schwarz.

Prueba DickeyFuller aumentada (ST)

Estadístico ADF

Valor crítico al $1 \%$

Valor crítico al 5\%

Valor crítico al $10 \%$

\section{$-1,397$}

$-3,769$

$-3,005$

$-2,642$

Nota: no se puede rechazar la hipótesis nula de la existencia de raíz unitaria.

Variable dependiente t-1

Estadístico $\mathrm{t}$

Constante

Estadístico $\mathrm{t}$

Número de rezagos

\section{$-0,022$}

$(-1,398)$

1110,699

$(4,061)$

0

Nota: si se incluye la tendencia, el coeficiente de la variable independiente cambia de signo y la serie se vuelve explosiva. El número de rezagos se determinó automáticamente por el criterio de selección Schwarz. 
Criterios de selección para obtener el rezago óptimo

\begin{tabular}{cccc}
\hline Número de rezagos & Akaike & Schwarz & Hannan-Quinn \\
\hline 0 & 40,121 & 40,220 & \\
1 & $34,083^{*}$ & $34,379^{*}$ & 34,135 \\
2 & 34,473 & 34,967 & 34,541 \\
3 & 34,763 & 35,456 & 34,859 \\
4 & 34,959 & 35,850 & 35,082 \\
5 & 34,768 & 35,856 & 34,918 \\
\hline
\end{tabular}

* Indica el rezago seleccionado según el criterio

Prueba Dickey Fuller aumentada [D(MI)]

\begin{tabular}{ll}
\hline Estadístico ADF & $-4,913$ \\
\hline & \\
\hline Valor crítico al 1\% & $-4,468$ \\
Valor crítico al 5\% & $-3,645$ \\
Valor crítico al 10\% & $-3,261$ \\
\hline
\end{tabular}

Nota: para todos los niveles de significancia se rechaza la hipótesis nula de existencia de raíz unitaria en las primeras diferencias.

\begin{tabular}{cc}
\hline Variable dependiente t-1 & $-1,151$ \\
Estadístico t & $(-4,913)$ \\
Constante & 241,141 \\
Estadístico t & $(0,169)$ \\
Tendencia & 238,478 \\
Estadístico $t$ & $(2,030)$ \\
Número de rezagos & 0 \\
\hline
\end{tabular}

Nota: El número de rezagos se determinó automáticamente por el criterio de selección Schwarz. 


\section{Prueba DickeyFuller aumentada [D(ST)]}

\begin{tabular}{lc}
\hline Estadístico ADF & $-3,196$ \\
& \\
Valor crítico al 1\% & $-4,468$ \\
Valor crítico al 5\% & $-3,645$ \\
Valor crítico al 10\% & $-3,261$ \\
\hline
\end{tabular}

Nota: No se puede rechazar la hipótesis nula de la existencia de raíz unitaria

$\begin{array}{cc}\text { Variable dependiente t- } 1 & -0,763 \\ \text { estadístico t } & (-3,196) \\ \text { Constante } & 832,840 \\ \text { estadístico t } & (2,757) \\ \text { Tendencia } & -22,005 \\ \text { Estadístico } t & (-1,419)\end{array}$

Número de rezagos 0

Nota: el número de rezagos se determinó automáticamente por el criterio de selección Schwarz.

Prueba Dickey Fuller aumentada [2D(ST)]

\begin{tabular}{cc}
\hline Estadístico ADF & $-5,368$ \\
\hline Valor crítico al 1\% & $-4,533$ \\
Valor crítico al 5\% & $-3,674$ \\
Valor crítico al 10\% & $-3,277$ \\
\hline Nota: para todos los niveles de significancia se rechaza la hipótesis nula de existencia de raíz unitaria en \\
& las segundas diferencias. \\
\hline Variable dependiente t-1 & $-2,001$ \\
Estadístico t & $(-5,368)$ \\
Constante & 202,813 \\
Estadístico t & $(0,734)$ \\
Tendencia & $-20,656$ \\
Estadístico $t$ & $(-1,049)$ \\
Número de rezagos & 1 \\
\hline
\end{tabular}

Nota: el número de rezagos se determinó automáticamente por el criterio de selección Schwarz.

tiempo\&economía

Vol. $3 \mathrm{~N}^{\circ} 1$ - I semestre de 2016 